TRANSACTIONS OF THE

AMERICAN MATHEMATICAL SOCIETY

Volume 363, Number 10, October 2011, Pages 5207-5222

S 0002-9947(2011)05202-5

Article electronically published on May 18, 2011

\title{
PARABOLIC MANIFOLDS FOR SEMI-ATTRACTIVE ANALYTIC TRANSFORMATIONS OF $\mathbf{C}^{n}$
}

\author{
FENG RONG
}

\begin{abstract}
We study the local dynamics of semi-attractive analytic transformations of $\mathbf{C}^{n}$. Under certain assumptions, Rivi showed the existence of parabolic manifolds of dimension $m+1$, where $m$ is the number of eigenvalues with modulus strictly less than one. Assuming moreover that certain matrix has $p$ eigenvalues with positive real part, we show the existence of parabolic manifolds of dimension $m+p+1$.
\end{abstract}

\section{INTRODUCTION}

Let $\Phi$ be an analytic transformation of $\mathbf{C}^{n}$ with a fixed point $p$. We say that $\Phi$ is semi-attractive at $p$ if $d \Phi_{p}=\operatorname{Diag}\left(I_{l}, \Lambda_{m}\right), l+m=n$, where the eigenvalues $\left\{\lambda_{i}\right\}_{1 \leq i \leq m}$ of $\Lambda_{m}$ have modulus strictly less than one. The local dynamics of such transformations have been studied by several authors ([F], [U1, [U2], [H1, Ri], [N]).

A preliminary investigation of semi-attractive analytic transformations of $\mathbf{C}^{2}$ was carried out by Fatou ([F]). Much later, Ueda ([U1, [U2]) studied the two dimensional case in greater detail. The higher dimensional case was first studied by Hakim ([H1] $)$, for $l=1$, and then by Rivi ([Ri] $)$, for $l>1$. While the above mentioned works focus on the dynamics tangent to the identity, the work of Nishimura ([N]) concerns more about the dynamics in the attracting directions.

In suitable local coordinates $(w, z) \in \mathbf{C}^{l} \times \mathbf{C}^{m}$, we can write $\Phi$ as

$$
\left\{\begin{aligned}
w_{1} & =f(w, z)=w+P_{1, z}(w)+P_{2, z}(w)+\cdots \\
z_{1} & =g(w, z)=C(z)+Q(w, z) w
\end{aligned}\right.
$$

where $P_{i, z}(w)$ are homogeneous polynomials in $w$ of degree $i$ whose coefficients are holomorphic functions in $z, C(z)$ is an analytic transformation of $\mathbf{C}^{m}$ with $C(0)=0$ and $d C(0)=\Lambda_{m}$, and $Q(w, z)$ is an $l \times m$ matrix whose elements are holomorphic functions of $\mathbf{C}^{n}$ vanishing at $(0,0)$.

We will always assume that $f(w, 0) \neq w$.

Let $f(w, 0)=w+P_{2}(w)+P_{3}(w)+\cdots$ be the homogeneous expansion of $f(w, 0)$. We say that $\Phi$ is of order $\nu$ if $P_{i}(w) \equiv 0$ for $i<\nu$ and $P_{\nu}(w) \not \equiv 0$. Consider $[v]=\left[v_{1}: \cdots: v_{n}\right] \in \mathbf{P}^{n-1}$ with $v_{i}=0, l<i \leq n$, and write $[v]=[\tilde{v}: 0]$. We say

Received by the editors August 8, 2008 and, in revised form, June 18, 2009.

2010 Mathematics Subject Classification. Primary 32H50.

(C)2011 American Mathematical Society Reverts to public domain 28 years from publication 5207 
that $[v]$ is a characteristic direction of $\Phi$ if $P_{\nu}(\tilde{v})=\lambda \tilde{v}$ for some $\lambda \in \mathbf{C}$. If $\lambda \neq 0$, then $[v]$ is said to be non-degenerate. Let $\hat{P}_{\nu}$ be the projection $\hat{P}_{\nu}:[X] \rightarrow\left[P_{\nu}(X)\right]$. The matrix associated to the linear map $\frac{1}{\nu-1}\left(d \hat{P}_{\nu}[\tilde{v}]-i d\right): T_{[\tilde{v}]} \mathbf{P}^{l-1} \rightarrow T_{[\tilde{v}]} \mathbf{P}^{l-1}$ is called the matrix associated to $[v]$.

A parabolic manifold of dimension $d$ for $\Phi$ at $p$ is an injective holomorphic map $\varphi: \Delta \rightarrow \mathbf{C}^{n}$ satisfying the following properties:

(i) $\Delta$ is a simply connected domain in $\mathbf{C}^{d}$ with $0 \in \partial \Delta$;

(ii) $\varphi$ is continuous on $\partial \Delta$ and $\varphi(0)=p$;

(iii) $\varphi(\Delta)$ is invariant under $\Phi$ and $\Phi^{k}(\varphi(\zeta)) \rightarrow p$ as $k \rightarrow \infty$ for any $\zeta \in \Delta$.

Furthermore, if $[\varphi(\zeta)] \rightarrow[v] \in \mathbf{P}^{n-1}$ as $\zeta \rightarrow 0$ (where [.] denotes the canonical projection of $\mathbf{C}^{n} \backslash\{p\}$ onto $\mathbf{P}^{n-1}$ ), we say that $\varphi$ is tangent to $[v]$ at $p$.

In [Ri], Rivi proved the following

Theorem 1.1. Let $\Phi$ be an analytic transformation of $\mathbf{C}^{n}$ with a semi-attractive fixed point $p$. Assume that $\Phi$ is of finite order $\nu$ and that $[v] \in \mathbf{P}^{n-1}$ is a nondegenerate characteristic direction of $\Phi$. Then there exists at least $\nu-1$ parabolic manifolds of dimension $m+1$, tangent to $\mathbf{C} v \oplus E$ at $p$, where $E$ is the generalized eigenspace associated to the eigenvalues of $d \Phi_{p}$ with modulus strictly less than one.

Our main result is the following

Theorem 1.2. Let $\Phi$ be an analytic transformation of $\mathbf{C}^{n}$ with a semi-attractive fixed point $p$. Assume that $\Phi$ is of finite order $\nu$ and that $[v] \in \mathbf{P}^{n-1}$ is a nondegenerate characteristic direction of $\Phi$. Let $\Gamma$ be the matrix associated to $[v]$. Assume that we can divide the eigenvalues of $\Gamma$ into two sets $\left\{\alpha_{j}\right\}_{1 \leq j \leq p}$ and $\left\{\beta_{k}\right\}_{1 \leq k \leq q}$ such that for some positive real number $\alpha$, we have

$$
\begin{array}{ll}
\operatorname{Re} \alpha_{j}>\alpha>0, & j=1, \cdots, p, \\
\operatorname{Re} \beta_{k}<\alpha, & k=1, \cdots, q .
\end{array}
$$

Then there exist at least $\nu-1$ parabolic manifolds of dimension $m+p+1$, tangent to $\mathbf{C} v \oplus E_{1} \oplus E_{2}$ at $p$, where $E_{1}$ is the generalized eigenspace associated to $\alpha_{j}$ 's and $E_{2}$ is the the generalized eigenspace associated to the eigenvalues of $d \Phi_{p}$ with modulus strictly less than one.

A similar result for so-called "quasi-parabolic" analytic transformations of $\mathbf{C}^{n}$ has been obtained in [Ro].

In Section 2, we recall some known results and define the operator $T$, which is needed in the proof of our main theorem. We then prove Theorem 1.2 in Section 3 ,

The author would like to thank the referee for many useful comments.

\section{The OPERATOR $T$}

Let $\Phi$ be as in (1.1). Assume that $\Phi$ is of finite order $\nu$. By [Ri, Proposition $2.2]$, for any $\mu>\nu$, we can choose local coordinates such that $\Phi$ takes the form

$$
\left\{\begin{array}{l}
w_{1}=f(w, z)=w+P_{\nu}(w)+\cdots+P_{\mu-1}(w)+P_{\mu, z}(w)+\cdots \\
z_{1}=g(w, z)=C(z)+Q(w, z) w
\end{array}\right.
$$

where $P_{i}(w)$ (resp. $\left.P_{i, z}(w)\right)$ are homogeneous polynomials in $w$ of degree $i$ with constant (resp. holomorphic functions in $z$ ) coefficients. 
Assume that $\Phi$ has a non-degenerate characteristic direction $[v]$. Up to a linear change of coordinates, we will assume that $[v]=[1: 0: \cdots: 0]$. Write $w=(x, y) \in$ $\mathbf{C} \times \mathbf{C}^{l-1}$ and set $P_{i}(x, y)=\left(p_{i}(x, y), q_{i}(x, y)\right)$ and $P_{i, z}(x, y)=\left(p_{i, z}(x, y), q_{i, z}(x, y)\right)$. Making the blow-up $y=\zeta x$ with $\zeta \in \mathbf{C}^{l-1}$, we have

$$
\left\{\begin{array}{l}
x_{1}=x+p_{\nu}(1, \zeta) x^{\nu}+\cdots+p_{\mu-1}(1, \zeta) x^{\mu-1}+p_{\mu, z}(1, \zeta) x^{\mu}+\cdots \\
\zeta_{1}=\zeta+r(\zeta) x^{\nu-1}+O\left(x^{\nu}\right)+O_{z}\left(x^{\mu-1}\right) \\
z_{1}=C(z)+O\left(x^{2},\|z\| x\right)
\end{array}\right.
$$

where $r(\zeta)=q_{\nu}(1, \zeta)-p_{\nu}(1, \zeta) \zeta$ and $O_{z}(h)=O(\|z\| h)$.

After replacing $r(\zeta)$ by its power series expansion at 0 and changing $x$ into $\lambda x$ with $\lambda^{\nu-1}=-p_{\nu}(1,0)(\nu-1)$, we have

$$
\left\{\begin{array}{l}
x_{1}=x-\frac{1}{\nu-1} x^{\nu}+O\left(x^{\nu+1},\|\zeta\| x^{\nu}\right)+O_{z}\left(x^{\mu}\right), \\
\zeta_{1}=\left(I-\Gamma x^{\nu-1}\right) \zeta+O\left(x^{\nu},\|\zeta\| x^{\nu},\|\zeta\|^{2} x^{\nu-1}\right)+O_{z}\left(x^{\mu-1}\right), \\
z_{1}=C(z)+O\left(x^{2},\|z\| x\right),
\end{array}\right.
$$

where $\Gamma=r^{\prime}(0) /\left((\nu-1) p_{\nu}(1,0)\right)$ is the matrix associated to $[v]$.

By [Ri, Proposition 3.1], the class of similarity of the matrix $\Gamma$ is invariant under changes of coordinates. Therefore, the eigenvalues of $\Gamma$ are invariants associated to $[v]$. We will assume, without loss of generality, that $\Gamma$ is in almost diagonal Jordan form.

For simplicity, from now on we will work with $\nu=2$. (One can deal with $\nu>2$ similarly; cf. [H3, Section 6].)

For $r, \rho, \tau$ small enough, define

$$
\Delta_{r, \rho, \tau}=\left\{(x, \zeta, z) \in \mathbf{C} \times \mathbf{C}^{l} \times \mathbf{C}^{m}:|x-r|<r,\|\zeta\|<\rho,\|z\|<\tau\right\} .
$$

Choose local coordinates in $\Delta_{r, \rho, \tau}$ such that the parabolic manifold provided by Theorem 1.1 is given by $\{\zeta=0\}$. We can then rewrite $\Phi$ as

$$
\left\{\begin{array}{l}
x_{1}=x-x^{2}+O_{1}\left(x^{3}\right)+O\left(\|\zeta\| x^{2}\right), \\
\zeta_{1}=(I-\Gamma x) \zeta+O_{1}\left(\|\zeta\| x^{2}\right)+O\left(\|\zeta\|^{2} x\right), \\
z_{1}=C(z)+O\left(x^{2},\|z\| x\right),
\end{array}\right.
$$

where $O_{1}(h)=O\left(h|\log x|^{q}\right)$ for some integer $q \geq 0$. The $\log x$ terms are introduced in some changes of coordinates needed in the proof of Theorem 1.1] (see [Ri] for details). The $\log x$ terms are well-defined, since we will only work in $\Delta_{r, \rho, \tau}$.

Divide the eigenvalues of $\Gamma$ as in (1.2). Write $\zeta=(u, v) \in \mathbf{C}^{p} \times \mathbf{C}^{q}$ and set $\Gamma=\operatorname{Diag}(A, B)$. We then have

$$
\left\{\begin{array}{l}
x_{1}=f(x, u, v, z)=x-x^{2}+F(x, u, v, z), \\
u_{1}=\psi(x, u, v, z)=\left(I_{p}-A x\right) u+G(x, u, v, z), \\
v_{1}=\phi(x, u, v, z)=\left(I_{q}-B x\right) v+H(x, u, v, z), \\
z_{1}=g(x, u, v, z)=C(z)+I(x, u, v, z),
\end{array}\right.
$$

where $F(x, u, v, z)=O_{1}\left(x^{3}\right)+O\left(\|\zeta\| x^{2}\right), G(x, u, v, z)=O_{1}\left(\|\zeta\| x^{2}\right)+O\left(\|\zeta\|^{2} x\right)$, $H(x, u, v, z)=O_{1}\left(\|\zeta\| x^{2}\right)+O\left(\|\zeta\|^{2} x\right)$ and $I(x, u, v, z)=O\left(x^{2},\|z\| x\right)$. 
We need the following

Proposition 2.1. Let $\Phi$ be as in (2.1). For every integer $m, k \geq 2$, we can choose coordinates in $\Delta_{r, \rho, \tau}$ such that

$$
H(x, u, 0, z)=O\left(\|u\|^{m} x,\|u\| x^{k}\right) .
$$

Proof. For terms involving only $x$ and $u$, we can argue as in [H3, Proposition 2.2].

Now, let $a_{s, t, j}(x, u, z)=p(z) x^{s}(\log x)^{t} u^{j}$ be a term involving $z$ in the expression of $H(x, u, 0, z)$, with $p(0)=0$. One readily checks that the transformation

$$
\tilde{v}=v+q(z) x^{s}(\log x)^{t} u^{j}
$$

cancels $a_{s, t, j}(x, u, z)$ if $q(z)=\sum_{i=0}^{\infty} p\left(C^{i}(z)\right)$. Since $C$ is a contraction, the series converges in a neighborhood of 0 .

By the construction in the proof of Theorem 1.1, we know that for each $s$ there are only finitely many $x^{s}(\log x)^{t}$ occurring. Therefore, starting with the smallest $s$ and $|j|$, we can make successive transformations as above such that terms involving $z$ in the expression of $H(x, u, 0, z)$ satisfy $O\left(\|u\|^{m} x,\|u\| x^{k}\right)$.

For $\gamma, s, \rho, \tau$ small enough, define

$$
S_{\gamma, s, \rho, \tau}=\left\{(x, u, z) \in \mathbf{C} \times \mathbf{C}^{p} \times \mathbf{C}^{m}:|\operatorname{Im} x|<\gamma \operatorname{Re} x,|x|<s,\|u\|<\rho,\|z\|<\tau\right\} .
$$

The following proposition concerns the case $p=l$, i.e. all the eigenvalues of $\Gamma$ have positive real parts.

Proposition 2.2. Let $\Phi$ be as in (2.1), with $A=\Gamma$. Then every point of the sector $S_{\gamma, s, \rho, \tau}$, with $\gamma, s, \rho, \tau$ small enough, is attracted to the origin in the direction $[1: 0: 0]$, and $x_{n}$ and $z_{n}$ are $O(1 / n)$. Moreover, we have $\left|x_{n}\right| \leq \frac{2}{n}$ and

$$
\left\|u_{n}\right\|\left|x_{n}\right|^{-\alpha} \leq\|u\||x|^{-\alpha} \text {. }
$$

Proof. In [H1], Hakim showed that the transformation $x_{1}=x-x^{2}+F(x, 0, z)$ is conjugated to $\frac{1}{x_{1}}=\frac{1}{x}+1$ in a domain of the type $\left\{\operatorname{Re} \frac{1}{x}>R\right\} \times\{\|z\|<\tau\}$, for $R$ large enough and $\tau$ small enough. Therefore, we can choose local coordinates such that $x_{1}=\frac{x}{1+x}+\tilde{F}(x, u, z)$, with $\tilde{F}(x, u, z)=O\left(\|u\| x^{2}\right)$.

We can then proceed as in [H3, Proposition 2.6] and [Ri, Proposition 4.1].

As in [H3], we then have the following

Corollary 2.3. Let $\Phi$ be as in Proposition 2.2 . For $(x, u, z) \in S_{\gamma, s, \rho, \tau}$, with $\gamma, s, \rho, \tau$ small enough, we have

$$
\lim _{n \rightarrow \infty}\left\|u_{n}\right\|\left|x_{n}\right|^{-\alpha}=0
$$

and

$$
\sum_{n=0}^{\infty}\left|x_{n}\right|^{t}\left|\log x_{n}\right|^{q} \leq C_{t, q}|x|^{t-1}|\log | x||^{q},
$$

where $t>1, q \in \mathbf{N}$ and $C_{t, q}$ is a constant.

Now, let $\Phi$ be as in (2.1), with $A \neq \Gamma$. We want to show that there exists a function $v=\varphi(x, u, z)$ in some sector $S_{\gamma, s, \rho, \tau}$ such that for

$$
\left\{\begin{array}{l}
x_{1}=f(x, u, \varphi(x, u, z), z) \\
u_{1}=\psi(x, u, \varphi(x, u, z), z) \\
z_{1}=g(x, u, \varphi(x, u, z), z)
\end{array}\right.
$$


we have

$$
\varphi\left(x_{1}, u_{1}, z_{1}\right)=\phi(x, u, \varphi(x, u, z), z) .
$$

Set $H_{1}(x, u, v, z)=v-x^{B} x_{1}^{-B} v_{1}$. One readily checks that $H_{1}$ satisfies the same estimates as $H$ :

$$
H_{1}(x, u, v, z)=O_{1}\left(\|\zeta\| x^{2}\right)+O\left(\|\zeta\|^{2} x\right)
$$

and

$$
H_{1}(x, u, 0, z)=O\left(\|u\|^{m} x,\|u\| x^{k}\right) .
$$

Also, the invariance (2.5) of $\varphi$ is equivalent to

$$
x^{-B} \varphi(x, u, z)-x_{1}^{-B} \varphi\left(x_{1}, u_{1}, z_{1}\right)=x^{-B} H_{1}(x, u, \varphi(x, u, z), z) .
$$

Let $\left\{\left(x_{n}, u_{n}, z_{n}\right)\right\}$ be the iterates of the transformation

$$
\left\{\begin{array}{l}
x_{1}=f(x, u, \varphi(x, u, z), z)=x-x^{2}+F(x, u, \varphi(x, u, z), z), \\
u_{1}=\psi(x, u, \varphi(x, u, z), z)=\left(I_{p}-A x\right) u+G(x, u, \varphi(x, u, z), z), \\
z_{1}=g(x, u, \varphi(x, u, z), z)=C(z)+I(x, u, \varphi(x, u, z), z),
\end{array}\right.
$$

with $f, \psi, g$ given in (2.1) and $\varphi$ analytic in some sector $S_{\gamma, s, \rho, \tau}$. Define

$$
T \varphi(x, u, z)=x^{B} \sum_{n=0}^{\infty} x_{n}^{-B} H_{1}\left(x_{n}, u_{n}, \varphi\left(x_{n}, u_{n}, z_{n}\right), z_{n}\right) .
$$

By Proposition 2.2 and Corollary 2.3 , we have the following proposition, whose proof is essentially the same as that of [H3, Proposition 3.2].

Proposition 2.4. Let $m, k$ be integers such that $H_{1}$ satisfies the estimate (2.7). There exists a positive constant $K_{0}$ such that

$$
\|\varphi(x, u, z)\| \leq K_{0}\left(\|u\|^{m}+\|u\| x^{k-1}\right)
$$

implies that the series (2.10) is uniformly converging on the invariant set

$$
D:=S_{\gamma, s, \rho, \tau} \cap\left\{\|u\||x|^{-\alpha} \leq 1\right\}
$$

and that

$$
\|T \varphi(x, u, z)\| \leq K_{0}\left(\|u\|^{m}+\|u\| x^{k-1}\right) .
$$

Let $B_{0}$ be the Banach space of holomorphic functions $\varphi$ on $D$ such that

$$
\|\varphi\|_{0}:=\sup _{x, u, z}\left\{\frac{|\varphi(x, u, z)|}{\|u\|^{m}+\|u\| x^{k-1}}\right\}
$$

is bounded, endowed with the norm $\|\varphi\|_{0}$. By Proposition 2.4, we have that $T$ is well-defined for $\varphi \in B_{0}$, with $T \varphi \in B_{0}$.

In the next section, we will prove the following

Proposition 2.5. There exists a closed subset $B_{1}$ of $B_{0}$ such that $T$ restricted to $B_{1}$ is a contraction. 
Proposition 2.5 gives us a fixed point of the operator $T$, which is a solution to (2.8), and thus to (2.5). Therefore, Theorem 1.2 follows from Proposition 2.5.

\section{Proof of Proposition 2.5}

First, let us fix some constants. Let $\delta=\min \{\alpha, 1\}$. Choose $\epsilon>0$ such that $\epsilon<\delta / 2$ and $\operatorname{Re} \alpha_{j}>\alpha+\epsilon$ for every eigenvalue $\alpha_{j}$ of $A$. Choose $\beta<\alpha$ such that $\operatorname{Re} \beta_{j}<\beta$ for every eigenvalue $\beta_{j}$ of $B$. (Throughout this section, $\|M\|$ denotes the Euclidean norm of the entries of $M$.)

Lemma 3.1. For $\gamma, s, \rho, \tau$ small enough, we have the following inequalities in $D$ :

$$
\begin{array}{llll}
\left|\frac{\partial x_{n}}{\partial x}\right| \leq\left|\frac{x_{n}}{x}\right|^{1+\delta-2 \epsilon}, & \left\|\frac{\partial x_{n}}{\partial u}\right\| \leq \frac{\left|x_{n}\right|^{1+\delta-2 \epsilon}}{|x|^{\delta-\epsilon}}, & & \left\|\frac{\partial x_{n}}{\partial z}\right\| \leq\left|x_{n}\right|^{1+\delta-2 \epsilon}, \\
\left\|\frac{\partial u_{n}}{\partial x}\right\| \leq \frac{\|u\|\left|x_{n}\right|^{\delta-\epsilon}}{|x|^{1+\delta-2 \epsilon}}, & \left\|\frac{\partial u_{n}}{\partial u}\right\| \leq\left|\frac{x_{n}}{x}\right|^{\delta-\epsilon}, & & \left\|\frac{\partial u_{n}}{\partial z}\right\| \leq\|u\|\left|x_{n}\right|^{\delta-\epsilon}, \\
\left\|\frac{\partial z_{n}}{\partial x}\right\| \leq 1, & \left\|\frac{\partial z_{n}}{\partial u}\right\| \leq 1, & \left\|\frac{\partial z_{n}}{\partial z}\right\| \leq 1 .
\end{array}
$$

Proof. First, note that given $b>a>0$ we have

$$
\left|x_{1}\right|^{a} \geq|x|^{a}|1-b x|
$$

for $|x|$ small enough.

For $\gamma, s, \rho, \tau$ small enough, we have the following inequalities in $D$ :

$$
\begin{aligned}
& \left|\frac{\partial x_{1}}{\partial x}\right|=|1-2 x+o(x)| \leq\left|\frac{x_{1}}{x}\right|^{2-\epsilon} \leq\left|\frac{x_{1}}{x}\right|^{1+\delta-2 \epsilon} \quad(1+\delta-\epsilon<2), \\
& \left\|\frac{\partial x_{1}}{\partial u}\right\| \leq K|x|^{2} \leq \frac{\left|x_{1}\right|^{1+\delta-2 \epsilon}}{|x|^{\delta-\epsilon}}, \\
& \left\|\frac{\partial x_{1}}{\partial z}\right\| \leq K\left(\left|x(\log x)^{q}\right|+\|u\|\right)|x|^{2} \leq|x|^{1-\delta+2 \epsilon}|x|^{1+\delta-2 \epsilon} \leq\left|x_{1}\right|^{1+\delta-2 \epsilon}, \\
& \left\|\frac{\partial u_{1}}{\partial x}\right\| \leq K\|u\| \leq \frac{\|u\|\left|x_{1}\right|^{\delta-\epsilon}}{|x|^{1+\delta-2 \epsilon}} \\
& \left\|\frac{\partial u_{1}}{\partial u}\right\| \leq|1-\alpha x| \leq\left|\frac{x_{1}}{x}\right|^{\delta-\epsilon} \quad(\delta-\epsilon<\alpha), \\
& \left\|\frac{\partial u_{1}}{\partial z}\right\| \leq K\left(\left|x(\log x)^{q}\right|+\|u\|\right)\|u\||x| \leq\|u\||x|^{1-\delta+\epsilon}|x|^{\delta-\epsilon} \leq\|u\|\left|x_{1}\right|^{\delta-\epsilon}, \\
& \left\|\frac{\partial z_{1}}{\partial x}\right\| \leq K(|x|+\|z\|) \leq 1, \\
& \left\|\frac{\partial z_{1}}{\partial u}\right\| \leq K\left(|x|^{2}+\|z\||x|\right) \leq 1 \\
& \left\|\frac{\partial z_{1}}{\partial z}\right\| \leq c+K|x| \leq 1 .
\end{aligned}
$$

Here $K$ is a constant independent of $x, u, v, z, q \in \mathbf{N}$ and $c<1$.

Therefore, (3.1) is true for $n=1$. The last row of (3.1) for an arbitrary $n$ is easily shown as above. We will show the other two rows of (3.1) by induction on $n$.

Assume that (3.1) is true for $n$. Using (2.3) and (3.2), we have

$$
\begin{aligned}
\left|\frac{\partial x_{n+1}}{\partial x}\right| \leq \mid 1-2 x_{n}+o\left(x_{n}\right) & \left.|| \frac{\partial x_{n}}{\partial x}|+K| x_{n}\right|^{2}\left\|\frac{\partial u_{n}}{\partial x}\right\| \\
& +K\left(\left|x_{n}\right|^{3}\left|\log x_{n}\right|^{q}+\left\|u_{n}\right\|\left|x_{n}\right|^{2}\right)\left\|\frac{\partial z_{n}}{\partial x}\right\|
\end{aligned}
$$




$$
\begin{aligned}
& \leq\left|\frac{x_{n}}{x}\right|^{1+\delta-2 \epsilon}\left(\left|1-2 x_{n}+o\left(x_{n}\right)\right|+\left.K\|u\||| x_{n}\right|^{1+\epsilon}\right. \\
& \left.+K\left(\left|x_{n}\right|^{2-\delta+2 \epsilon}\left|\log x_{n}\right|^{q}+\left\|u_{n}\right\|\left|x_{n}\right|^{1-\delta+2 \epsilon}\right)|x|^{1+\delta-2 \epsilon}\right) \\
& \leq\left|\frac{x_{n}}{x}\right|^{1+\delta-2 \epsilon}\left(\left|1-2 x_{n}+o\left(x_{n}\right)\right|+K\left|x_{n}\right|\left(\left|x_{n}\right|^{1-\delta+2 \epsilon}\left|\log x_{n}\right|^{q}\right.\right. \\
& \left.\left.+\|u\||x|^{-\alpha}\left|x_{n}\right|^{1-\delta+2 \epsilon+\alpha}\right)|x|^{1+\delta-2 \epsilon}\right) \\
& \leq\left|\frac{x_{n+1}}{x}\right|^{1+\delta-2 \epsilon}, \\
& \left\|\frac{\partial x_{n+1}}{\partial u}\right\| \leq\left|1-2 x_{n}+o\left(x_{n}\right)\right|\left\|\frac{\partial x_{n}}{\partial u}\right\|+K\left|x_{n}\right|^{2}\left\|\frac{\partial u_{n}}{\partial u}\right\| \\
& +K\left(\left|x_{n}\right|^{3}\left|\log x_{n}\right|^{q}+\left\|u_{n}\right\|\left|x_{n}\right|^{2}\right)\left\|\frac{\partial z_{n}}{\partial u}\right\| \\
& \leq \frac{\left|x_{n}\right|^{1+\delta-2 \epsilon}}{|x|^{\delta-\epsilon}}\left(\left|1-2 x_{n}+o\left(x_{n}\right)\right|+K\left|x_{n}\right|^{1+\epsilon}\right. \\
& \left.+K\left(\left|x_{n}\right|^{2-\delta+2 \epsilon}\left|\log x_{n}\right|^{q}+\left\|u_{n}\right\|\left|x_{n}\right|^{1-\delta+2 \epsilon}\right)|x|^{\delta-\epsilon}\right) \\
& \leq \frac{\left|x_{n+1}\right|^{1+\delta-2 \epsilon}}{|x|^{\delta-\epsilon}}, \\
& \left\|\frac{\partial x_{n+1}}{\partial z}\right\| \leq\left|1-2 x_{n}+o\left(x_{n}\right)\right|\left\|\frac{\partial x_{n}}{\partial z}\right\|+K\left|x_{n}\right|^{2}\left\|\frac{\partial u_{n}}{\partial z}\right\| \\
& +K\left(\left|x_{n}\right|^{3}\left|\log x_{n}\right|^{q}+\left\|u_{n}\right\|\left|x_{n}\right|^{2}\right)\left\|\frac{\partial z_{n}}{\partial z}\right\| \\
& \leq\left|x_{n}\right|^{1+\delta-2 \epsilon}\left(\left|1-2 x_{n}+o\left(x_{n}\right)\right|+\left.K\|u\||| x_{n}\right|^{1+\epsilon}\right. \\
& \left.+K\left(\left|x_{n}\right|^{2-\delta+2 \epsilon}\left|\log x_{n}\right|^{q}+\left\|u_{n}\right\|\left|x_{n}\right|^{1-\delta+2 \epsilon}\right)\right) \\
& \leq\left|x_{n+1}\right|^{1+\delta-2 \epsilon}, \\
& \left\|\frac{\partial u_{n+1}}{\partial x}\right\| \leq K\left\|u_{n}\right\|\left|\frac{\partial x_{n}}{\partial x}\right|+\left|1-\alpha x_{n}\right| \mid \frac{\partial u_{n}}{\partial x} \| \\
& +K\left(\left\|u_{n}\right\|\left|x_{n}\right|^{2}\left|\log x_{n}\right|^{q}+\left\|u_{n}\right\|^{2}\left|x_{n}\right|\right)\left\|\frac{\partial z_{n}}{\partial x}\right\| \\
& \leq \frac{\left.\|u\||| x_{n}\right|^{\delta-\epsilon}}{|x|^{1+\delta-2 \epsilon}}\left(\left|1-\alpha x_{n}\right|+K|x|^{-\alpha}\left|x_{n}\right|^{1-\epsilon+\alpha}\right. \\
& \left.+K\left(|x|^{-\alpha}\left|x_{n}\right|^{2-\delta+\epsilon+\alpha}\left|\log x_{n}\right|^{q}+\left.\|u\||| x\right|^{-2 \alpha}\left|x_{n}\right|^{1-\delta+\epsilon+2 \alpha}\right)|x|^{1+\delta-2 \epsilon}\right) \\
& \leq \frac{\|u\|\left|x_{n+1}\right|^{\delta-\epsilon}}{|x|^{1+\delta-2 \epsilon}} \\
& \left\|\frac{\partial u_{n+1}}{\partial u}\right\| \leq K\left\|u_{n}\right\|\left\|\frac{\partial x_{n}}{\partial u}\right\|+\left|1-\alpha x_{n}\right|\left\|\frac{\partial u_{n}}{\partial u}\right\| \\
& +K\left(\left\|u_{n}||\left|x_{n}\right|^{2}\left|\log x_{n}\right|^{q}+\right\| u_{n} \|^{2}\left|x_{n}\right|\right)\left\|\frac{\partial z_{n}}{\partial u}\right\| \\
& \leq\left|\frac{x_{n}}{x}\right|^{\delta-\epsilon}\left(\left|1-\alpha x_{n}\right|+\left.K\|u\||| x\right|^{-\alpha}\left|x_{n}\right|^{1-\epsilon+\alpha}\right. \\
& \left.+K\left(\|u\||x|^{-\alpha}\left|x_{n}\right|^{2-\delta+\epsilon+\alpha}\left|\log x_{n}\right|^{q}+\|u\|^{2}|x|^{-2 \alpha}\left|x_{n}\right|^{1-\delta+\epsilon+2 \alpha}\right)|x|^{\delta-\epsilon}\right) \\
& \leq\left|\frac{x_{n+1}}{x}\right|^{\delta-\epsilon},
\end{aligned}
$$




$$
\begin{aligned}
\left\|\frac{\partial u_{n+1}}{\partial z}\right\| \leq & K\left\|u_{n}\right\|\left\|\frac{\partial x_{n}}{\partial z}\right\|+\left|1-\alpha x_{n}\right|\left\|\frac{\partial u_{n}}{\partial z}\right\| \\
& \quad+K\left(\left\|u_{n}\right\|\left|x_{n}\right|^{2}\left|\log x_{n}\right|^{q}+\left\|u_{n}\right\|^{2}\left|x_{n}\right|\right)\left\|\frac{\partial z_{n}}{\partial z}\right\| \\
\leq & \left.\|u\||| x_{n}\right|^{\delta-\epsilon}\left(\left|1-\alpha x_{n}\right|+\left.K\|u\||| x\right|^{-\alpha}\left|x_{n}\right|^{1-\epsilon+\alpha}\right. \\
& \left.+K\left(|x|^{-\alpha}\left|x_{n}\right|^{2-\delta+\epsilon+\alpha}\left|\log x_{n}\right|^{q}+\|u\||x|^{-2 \alpha}\left|x_{n}\right|^{1-\delta+\epsilon+2 \alpha}\right)\right) \\
\leq & \left.\|u\||| x_{n+1}\right|^{\delta-\epsilon} .
\end{aligned}
$$

This proves the lemma.

Proposition 3.2. Let $\varphi$ be in $B_{0}$. There are positive constants $K_{1}, K_{2}$ and $K_{3}$ such that

$$
\left\{\begin{array}{l}
\left\|\frac{\partial \varphi}{\partial x}\right\| \leq K_{1}\left(\|u\|^{m}|x|^{-1}+\|u\||x|^{k-2}\right), \\
\left\|\frac{\partial \varphi}{\partial u}\right\| \leq K_{2}\left(\|u\|^{m-1}+|x|^{k-1}\right) \\
\left\|\frac{\partial \varphi}{\partial z}\right\| \leq K_{3}\left(\|u\|^{m}+\|u\||x|^{k-1}\right)
\end{array}\right.
$$

imply the same inequalities for $\left\|\frac{\partial T \varphi}{\partial x}\right\|,\left\|\frac{\partial T \varphi}{\partial u}\right\|$ and $\left\|\frac{\partial T \varphi}{\partial z}\right\|$.

Proof. Recall that

$$
T \varphi(x, u, z)=\sum_{n=0}^{\infty}\left(\frac{x_{n}}{x}\right)^{-B} H_{1}\left(x_{n}, u_{n}, \varphi\left(x_{n}, u_{n}, z_{n}\right), z_{n}\right) .
$$

Note that we have the following bounds for $H_{1}(x, u, \varphi(x, u, z), z)$, with $\varphi \in B_{0}$ :

$$
\begin{aligned}
&\left\|H_{1}(x, u, \varphi(x, u, z), z)\right\| \leq C_{1}\left(\|u\|^{m}|x|+\|u\||x|^{k}\right) \\
&+C_{2}\|\varphi\|\left(|x|^{2}|\log x|^{q}+\|\varphi\||x|+\|u\||x|\right) \\
& \leq\left(C_{1}+1\right)\left(\|u\|^{m}|x|+\|u\||x|^{k}\right),
\end{aligned}
$$

where $C_{1}$ and $C_{2}$ are constants depending only on $H_{1}$. Therefore, we have the following bounds for the partial derivatives of $H_{1}$ :

$$
\begin{aligned}
& \left\|\frac{\partial H_{1}}{\partial x}(x, u, \varphi(x, u, z), z)\right\| \leq C_{3}\left(\|u\|^{m}+\|u\||x|^{k-1}\right), \\
& \left\|\frac{\partial H_{1}}{\partial u}(x, u, \varphi(x, u, z), z)\right\| \leq C_{3}\left(\|u\|^{m-1}|x|+|x|^{k}\right), \\
& \left\|\frac{\partial H_{1}}{\partial z}(x, u, \varphi(x, u, z), z)\right\| \leq C_{3}\left(\|u\|^{m}|x|+\|u\||x|^{k}\right), \\
& \left\|\frac{\partial H_{1}}{\partial \varphi}(x, u, \varphi(x, u, z), z)\right\| \leq C_{4}\left(|x|^{2}|\log x|^{q}+\|u\||x|\right),
\end{aligned}
$$

where $C_{3}$ and $C_{4}$ are constants depending only on $H_{1}$.

Assume that the inequalities (3.3) are satisfied for arbitrarily given constants $K_{1}^{\prime}, K_{2}^{\prime}$ and $K_{3}^{\prime}$. 
Differentiating (3.4) with respect to $x$, we have

$$
\frac{\partial}{\partial x} T \varphi(x, u, z)=r_{1}+r_{2}+r_{3}+r_{4}+r_{5}+r_{6}
$$

where

$$
\begin{aligned}
& r_{1}=B x^{-1} \sum_{n=0}^{\infty}\left(\frac{x_{n}}{x}\right)^{-B} H_{1}\left(x_{n}, u_{n}, \varphi\left(x_{n}, u_{n}, z_{n}\right), z_{n}\right), \\
& r_{2}=-B \sum_{n=0}^{\infty}\left(\frac{x_{n}}{x}\right)^{-B} H_{1}\left(x_{n}, u_{n}, \varphi\left(x_{n}, u_{n}, z_{n}\right), z_{n}\right) x_{n}^{-1} \frac{\partial x_{n}}{\partial x}, \\
& r_{3}=\sum_{n=0}^{\infty}\left(\frac{x_{n}}{x}\right)^{-B} \frac{\partial}{\partial x_{n}} H_{1}\left(x_{n}, u_{n}, \varphi\left(x_{n}, u_{n}, z_{n}\right), z_{n}\right) \frac{\partial x_{n}}{\partial x}, \\
& r_{4}=\sum_{n=0}^{\infty}\left(\frac{x_{n}}{x}\right)^{-B} \frac{\partial}{\partial u_{n}} H_{1}\left(x_{n}, u_{n}, \varphi\left(x_{n}, u_{n}, z_{n}\right), z_{n}\right) \frac{\partial u_{n}}{\partial x}, \\
& r_{5}=\sum_{n=0}^{\infty}\left(\frac{x_{n}}{x}\right)^{-B} \frac{\partial}{\partial z_{n}} H_{1}\left(x_{n}, u_{n}, \varphi\left(x_{n}, u_{n}, z_{n}\right), z_{n}\right) \frac{\partial z_{n}}{\partial x}, \\
& r_{6}=\sum_{n=0}^{\infty}\left(\frac{x_{n}}{x}\right)^{-B} \frac{\partial}{\partial \varphi} H_{1}\left(x_{n}, u_{n}, \varphi\left(x_{n}, u_{n}, z_{n}\right), z_{n}\right)\left(\frac{\partial \varphi}{\partial x_{n}} \frac{\partial x_{n}}{\partial x}+\frac{\partial \varphi}{\partial u_{n}} \frac{\partial u_{n}}{\partial x}+\frac{\partial \varphi}{\partial z_{n}} \frac{\partial z_{n}}{\partial x}\right) .
\end{aligned}
$$

Using (2.3), (2.4), (3.5), 3.6) and Lemma 3.1, we have

$$
\begin{aligned}
\left\|r_{1}\right\| & \leq \beta|x|^{-1} \sum_{n=0}^{\infty}\left|\frac{x_{n}}{x}\right|^{-\beta}\left(C_{1}+1\right)\left(\left\|u_{n}\right\|^{m}\left|x_{n}\right|+\left\|u_{n}\right\|\left|x_{n}\right|^{k}\right) \\
& \leq \beta\left(C_{1}+1\right)|x|^{-1} \sum_{n=0}^{\infty}\left|\frac{x_{n}}{x}\right|^{-\beta}\left(\|u\|^{m}|x|^{-m \alpha}\left|x_{n}\right|^{m \alpha+1}+\|u\||x|^{-\alpha}\left|x_{n}\right|^{\alpha+k}\right) \\
& \leq C_{1}^{\prime}\left(\|u\|^{m}|x|^{-1}+\|u\||x|^{k-2}\right), \\
\left\|r_{2}\right\| & \leq \beta \sum_{n=0}^{\infty}\left|\frac{x_{n}}{x}\right|^{-\beta}\left(C_{1}+1\right)\left(\left\|u_{n}\right\|^{m}\left|x_{n}\right|+|| u_{n}||\left|x_{n}\right|^{k}\right)\left|x_{n}\right|^{-1}\left|\frac{x_{n}}{x}\right|^{1+\delta-2 \epsilon} \\
& \leq C_{2}^{\prime}\left(\|u\|^{m}|x|^{-1}+\|u\||x|^{k-2}\right), \\
\left\|r_{3}\right\| & \leq \sum_{n=0}^{\infty}\left|\frac{x_{n}}{x}\right|^{-\beta} C_{3}\left(\left\|u_{n}\right\|^{m}+\left\|u_{n}\right\|\left|x_{n}\right|^{k-1}\right)\left|\frac{x_{n}}{x}\right|^{1+\delta-2 \epsilon} \\
& \leq C_{3}^{\prime}\left(\|u\|^{m}|x|^{-1}+\|u\||x|^{k-2}\right), \\
\left\|r_{4}\right\| & \leq \sum_{n=0}^{\infty}\left|\frac{x_{n}}{x}\right|^{-\beta} C_{3}\left(\left\|u_{n}\right\|^{m-1}\left|x_{n}\right|+\left|x_{n}\right|^{k}\right) \frac{\|u\|\left|x_{n}\right|^{\delta-\epsilon}}{|x|^{1+\delta-2 \epsilon}} \\
& \leq C_{3} \sum_{n=0}^{\infty}\left|\frac{x_{n}}{x}\right|^{\delta-\epsilon-\beta}\left(\|u\|^{m}|x|^{-(m-1) \alpha}\left|x_{n}\right|^{(m-1) \alpha+1}+\|u\|\left|x_{n}\right|^{k}\right)|x|^{-1+\epsilon} \\
& \leq C_{4}^{\prime}\left(\|u\|^{m}|x|^{-1}+\|u\||x|^{k-2}\right), \\
\left\|r_{5}\right\| & \leq \sum_{n=0}^{\infty}\left|\frac{x_{n}}{x}\right|^{-\beta} C_{3}\left(\|u\|^{m}|x|^{-1}+\|u\||x|^{k-2}\right), \\
& \left.\leq\left\|_{n}^{m}\left|x_{n}\right|+\right\| u_{n} \|\left|x_{n}\right|^{k}\right)
\end{aligned}
$$




$$
\begin{gathered}
\left\|r_{6}\right\| \leq \sum_{n=0}^{\infty}\left|\frac{x_{n}}{x}\right|^{-\beta} C_{4}\left(\left|x_{n}\right|^{2}\left|\log x_{n}\right|^{q}+\left\|u_{n}\right\|\left|x_{n}\right|\right)\left(K_{3}^{\prime}\left(\left\|u_{n}\right\|^{m}+\left\|u_{n}\right\|\left|x_{n}\right|^{k-1}\right)\right. \\
+K_{1}^{\prime}\left(\left\|u_{n}\right\|^{m}\left|x_{n}\right|^{-1}+\left.\left\|u_{n}\right\||| x_{n}\right|^{k-2}\right)\left|\frac{x_{n}}{x}\right|^{1+\delta-2 \epsilon} \\
\left.+K_{2}^{\prime}\left(\left\|u_{n}\right\|^{m-1}+\left|x_{n}\right|^{k-1}\right) \frac{\left.\|u\||| x_{n}\right|^{\delta-\epsilon}}{|x|^{1+\delta-2 \epsilon}}\right) \\
\leq C_{4} \sum_{n=0}^{\infty}\left|\frac{x_{n}}{x}\right|^{-\beta}\left(\left\|u_{n}\right\|^{m-1}+\left|x_{n}\right|^{k-1}\right)\left(K_{3}^{\prime}\left(\left.\left\|u_{n}\right\||| x_{n}\right|^{2}\left|\log x_{n}\right|^{q}+\left\|u_{n}\right\|^{2}\left|x_{n}\right|\right)\right. \\
+K_{1}^{\prime}\left(\left\|u_{n}\right\|\left|x_{n}\right|\left|\log x_{n}\right|^{q}+\left\|u_{n}\right\|^{2}\right)\left|\frac{x_{n}}{x}\right|^{1+\delta-2 \epsilon} \\
\left.+K_{2}^{\prime}\left(\left|x_{n}\right|^{2}\left|\log x_{n}\right|^{q}+\left\|u_{n}\right\|\left|x_{n}\right|\right) \frac{|| u \|\left. x_{n}\right|^{\delta-\epsilon}}{|x|^{1+\delta-2 \epsilon}}\right) \\
\leq C_{6}^{\prime}\left(\|u\|^{m}|x|^{-1}+\|u\||x|^{k-2}\right),
\end{gathered}
$$

where $C_{1}^{\prime}, C_{2}^{\prime}, C_{3}^{\prime}, C_{4}^{\prime}$ are constants depending only on $H_{1}$ and where $C_{5}^{\prime}, C_{6}^{\prime}$ are small compared to $C_{j}^{\prime}, 1 \leq j \leq 4$. Set

$$
K_{1}=2\left(C_{1}^{\prime}+C_{2}^{\prime}+C_{3}^{\prime}+C_{4}^{\prime}\right) .
$$

We then have

$$
\left\|\frac{\partial T \varphi}{\partial x}\right\| \leq K_{1}\left(\|u\|^{m}|x|^{-1}+\|u\||x|^{k-2}\right) .
$$

Differentiating (3.4) with respect to $u$, we have

$$
\frac{\partial}{\partial u} T \varphi(x, u, z)=s_{1}+s_{2}+s_{3}+s_{4}+s_{5},
$$

where

$$
\begin{aligned}
& s_{1}=-B \sum_{n=0}^{\infty}\left(\frac{x_{n}}{x}\right)^{-B} H_{1}\left(x_{n}, u_{n}, \varphi\left(x_{n}, u_{n}, z_{n}\right), z_{n}\right) x_{n}^{-1} \frac{\partial x_{n}}{\partial u}, \\
& s_{2}=\sum_{n=0}^{\infty}\left(\frac{x_{n}}{x}\right)^{-B} \frac{\partial}{\partial x_{n}} H_{1}\left(x_{n}, u_{n}, \varphi\left(x_{n}, u_{n}, z_{n}\right), z_{n}\right) \frac{\partial x_{n}}{\partial u}, \\
& s_{3}=\sum_{n=0}^{\infty}\left(\frac{x_{n}}{x}\right)^{-B} \frac{\partial}{\partial u_{n}} H_{1}\left(x_{n}, u_{n}, \varphi\left(x_{n}, u_{n}, z_{n}\right), z_{n}\right) \frac{\partial u_{n}}{\partial u}, \\
& s_{4}=\sum_{n=0}^{\infty}\left(\frac{x_{n}}{x}\right)^{-B} \frac{\partial}{\partial z_{n}} H_{1}\left(x_{n}, u_{n}, \varphi\left(x_{n}, u_{n}, z_{n}\right), z_{n}\right) \frac{\partial z_{n}}{\partial u}, \\
& s_{5}=\sum_{n=0}^{\infty}\left(\frac{x_{n}}{x}\right)^{-B} \frac{\partial}{\partial \varphi} H_{1}\left(x_{n}, u_{n}, \varphi\left(x_{n}, u_{n}, z_{n}\right), z_{n}\right)\left(\frac{\partial \varphi}{\partial x_{n}} \frac{\partial x_{n}}{\partial u}+\frac{\partial \varphi}{\partial u_{n}} \frac{\partial u_{n}}{\partial u}+\frac{\partial \varphi}{\partial z_{n}} \frac{\partial z_{n}}{\partial u}\right) .
\end{aligned}
$$

Using (2.3), (2.4), (3.5), (3.6) and Lemma 3.1, we have

$$
\begin{aligned}
\left\|s_{1}\right\| & \leq \beta \sum_{n=0}^{\infty}\left|\frac{x_{n}}{x}\right|^{-\beta}\left(C_{1}+1\right)\left(\left\|u_{n}\right\|^{m}\left|x_{n}\right|+\left\|u_{n}\right\|\left|x_{n}\right|^{k}\right)\left|x_{n}\right|^{-1} \frac{\left|x_{n}\right|^{1+\delta-2 \epsilon}}{|x|^{\delta-\epsilon}} \\
& \leq \beta\left(C_{1}+1\right) \sum_{n=0}^{\infty}\left|\frac{x_{n}}{x}\right|^{\delta-\epsilon-\beta}\left(\|u\|^{m}|x|^{-m \alpha}\left|x_{n}\right|^{m \alpha+1}+\left.\|u\||| x\right|^{-\alpha}\left|x_{n}\right|^{\alpha+k}\right)\left|x_{n}\right|^{-\epsilon}
\end{aligned}
$$




$$
\begin{aligned}
& \leq C_{1}^{\prime \prime}\left(\|u\|^{m-1}+|x|^{k-1}\right), \\
\left\|s_{2}\right\| & \leq \sum_{n=0}^{\infty}\left|\frac{x_{n}}{x}\right|^{-\beta} C_{3}\left(\left\|u_{n}\right\|^{m}+\left\|\left.u_{n}|\|| x_{n}\right|^{k-1}\right) \frac{\left|x_{n}\right|^{1+\delta-2 \epsilon}}{|x|^{\delta-\epsilon}}\right. \\
& \leq\left. C_{3} \sum_{n=0}^{\infty}\left|\frac{x_{n}}{x}\right|^{\delta-\epsilon-\beta}\left(\|u\|^{m-1}|x|^{-m \alpha}\left|x_{n}\right|^{m \alpha+1}+|x|^{-\alpha}\left|x_{n}\right|^{\alpha+k}\right)\|u\||| x_{n}\right|^{-\epsilon} \\
& \leq C_{2}^{\prime \prime}\left(\|u\|^{m-1}+|x|^{k-1}\right), \\
\left\|s_{3}\right\| & \leq \sum_{n=0}^{\infty}\left|\frac{x_{n}}{x}\right|^{-\beta} C_{3}\left(\left\|u_{n}\right\|^{m-1}\left|x_{n}\right|+\left|x_{n}\right|^{k}\right)\left|\frac{x_{n}}{x}\right|^{\delta-\epsilon} \\
& \leq C_{3}^{\prime \prime}\left(\|u\|^{m-1}+|x|^{k-1}\right), \\
\left\|s_{4}\right\| & \leq \sum_{n=0}^{\infty}\left|\frac{x_{n}}{x}\right|^{-\beta} C_{3}\left(\left\|u_{n}\right\|^{m}\left|x_{n}\right|+\left\|u_{n}\right\|\left|x_{n}\right|^{k}\right) \\
& \leq C_{4}^{\prime \prime}\left(\|u\|^{m-1}+|x|^{k-1}\right), \\
\left\|s_{5}\right\| & \leq \sum_{n=0}^{\infty}\left|\frac{x_{n}}{x}\right|^{-\beta} C_{4}\left(\left|x_{n}\right|^{2}\left|\log x_{n}\right|^{q}+\left\|u_{n}\right\|\left|x_{n}\right|\right)\left(K_{3}^{\prime}\left(\left\|u_{n}\right\|^{m}+\left\|u_{n}\right\|\left|x_{n}\right|^{k-1}\right)\right) \\
& \quad+K_{5}^{\prime \prime}\left(\left\|u_{n}\right\|^{m}\left|x_{n}\right|^{-1}+\|u\|^{m-1}+|x|^{k-1}\right), \\
& \quad+K_{2}^{\prime}\left(\left\|u_{n}\right\|^{m-1}+\left|x_{n}\right|^{k-2}\right) \frac{\left|x_{n}\right|^{1+\delta-2 \epsilon}}{|x|^{\delta-\epsilon}} \\
& \leq C_{4} \sum_{n=0}^{\infty}\left|\frac{x_{n}}{x}\right|^{-\beta}\left(\left\|u_{n}\right\|^{m-1}+\left|x_{n}\right|^{k-1}\right)\left(K _ { 3 } ^ { \prime } \left(\left\|u_{n}\left|\left\|\left|x_{n}\right|^{2}\left|\log x_{n}\right|^{q}+\right\| u_{n} \|^{2}\right| x_{n} \mid\right)\right.\right. \\
& +K_{1}^{\prime}\left(\left\|u_{n}\right\|\left|x_{n}\left\|\left.\log x_{n}\right|^{q}+\right\| u_{n} \|^{2}\right) \frac{\left|x_{n}\right|^{1+\delta-2 \epsilon}}{|x|^{\delta-\epsilon}}\right.
\end{aligned}
$$

where $C_{1}^{\prime \prime}, C_{2}^{\prime \prime}, C_{3}^{\prime \prime}$ are constants depending only on $H_{1}$ and where $C_{4}^{\prime \prime}, C_{5}^{\prime \prime}$ are small compared to $C_{j}^{\prime \prime}, 1 \leq j \leq 3$. Set

$$
K_{2}=2\left(C_{1}^{\prime \prime}+C_{2}^{\prime \prime}+C_{3}^{\prime \prime}\right) .
$$

We then have

$$
\left\|\frac{\partial T \varphi}{\partial u}\right\| \leq K_{2}\left(\|u\|^{m-1}+|x|^{k-1}\right) .
$$

Differentiating (3.4) with respect to $z$, we have

$$
\frac{\partial}{\partial z} T \varphi(x, u, z)=t_{1}+t_{2}+t_{3}+t_{4}+t_{5}
$$

where

$$
t_{1}=-B \sum_{n=0}^{\infty}\left(\frac{x_{n}}{x}\right)^{-B} H_{1}\left(x_{n}, u_{n}, \varphi\left(x_{n}, u_{n}, z_{n}\right), z_{n}\right) x_{n}^{-1} \frac{\partial x_{n}}{\partial z},
$$




$$
\begin{aligned}
t_{2} & =\sum_{n=0}^{\infty}\left(\frac{x_{n}}{x}\right)^{-B} \frac{\partial}{\partial x_{n}} H_{1}\left(x_{n}, u_{n}, \varphi\left(x_{n}, u_{n}, z_{n}\right), z_{n}\right) \frac{\partial x_{n}}{\partial z}, \\
t_{3} & =\sum_{n=0}^{\infty}\left(\frac{x_{n}}{x}\right)^{-B} \frac{\partial}{\partial u_{n}} H_{1}\left(x_{n}, u_{n}, \varphi\left(x_{n}, u_{n}, z_{n}\right), z_{n}\right) \frac{\partial u_{n}}{\partial z}, \\
t_{4} & =\sum_{n=0}^{\infty}\left(\frac{x_{n}}{x}\right)^{-B} \frac{\partial}{\partial z_{n}} H_{1}\left(x_{n}, u_{n}, \varphi\left(x_{n}, u_{n}, z_{n}\right), z_{n}\right) \frac{\partial z_{n}}{\partial z}, \\
t_{5} & =\sum_{n=0}^{\infty}\left(\frac{x_{n}}{x}\right)^{-B} \frac{\partial}{\partial \varphi} H_{1}\left(x_{n}, u_{n}, \varphi\left(x_{n}, u_{n}, z_{n}\right), z_{n}\right)\left(\frac{\partial \varphi}{\partial x_{n}} \frac{\partial x_{n}}{\partial z}+\frac{\partial \varphi}{\partial u_{n}} \frac{\partial u_{n}}{\partial z}+\frac{\partial \varphi}{\partial z_{n}} \frac{\partial z_{n}}{\partial z}\right) .
\end{aligned}
$$

Using (2.3), (2.4), (3.5), (3.6) and Lemma 3.1, we have

$$
\begin{aligned}
& \left\|t_{1}\right\| \leq \beta \sum_{n=0}^{\infty}\left|\frac{x_{n}}{x}\right|^{-\beta}\left(C_{1}+1\right)\left(\left\|u_{n}\right\|^{m}\left|x_{n}\right|+\| u_{n}||\left|x_{n}\right|^{k}\right)\left|x_{n}\right|^{\delta-2 \epsilon} \\
& \leq C_{1}^{\prime \prime \prime}\left(\|u\|^{m}+\|u\||x|^{k-1}\right), \\
& \left\|t_{2}\right\| \leq \sum_{n=0}^{\infty}\left|\frac{x_{n}}{x}\right|^{-\beta} C_{3}\left(\left\|u_{n}\right\|^{m}+\left\|u_{n}\right\|\left|x_{n}\right|^{k-1}\right)\left|x_{n}\right|^{1+\delta-2 \epsilon} \\
& \leq C_{2}^{\prime \prime \prime}\left(\|u\|^{m}+\|u\||x|^{k-1}\right), \\
& \left\|t_{3}\right\| \leq \sum_{n=0}^{\infty}\left|\frac{x_{n}}{x}\right|^{-\beta} C_{3}\left(\left\|u_{n}\right\|^{m-1}\left|x_{n}\right|+\left|x_{n}\right|^{k}\right)\|u\|\left|x_{n}\right|^{\delta-\epsilon} \\
& \leq C_{3}^{\prime \prime \prime}\left(\|u\|^{m}+\|u\||x|^{k-1}\right), \\
& \left\|t_{4}\right\| \leq \sum_{n=0}^{\infty}\left|\frac{x_{n}}{x}\right|^{-\beta} C_{3}\left(\left\|u_{n}\right\|^{m}\left|x_{n}\right|+\left\|u_{n}\right\|\left|x_{n}\right|^{k}\right) \\
& \leq C_{4}^{\prime \prime \prime}\left(\|u\|^{m}+\|u\||x|^{k-1}\right), \\
& \left\|t_{5}\right\| \leq \sum_{n=0}^{\infty}\left|\frac{x_{n}}{x}\right|^{-\beta} C_{4}\left(\left|x_{n}\right|^{2}\left|\log x_{n}\right|^{q}+\left\|u_{n}\right\|\left|x_{n}\right|\right)\left(K_{3}^{\prime}\left(\left\|u_{n}\right\|^{m}+\left\|u_{n}\right\|\left|x_{n}\right|^{k-1}\right)\right) \\
& +K_{1}^{\prime}\left(\left\|u_{n}\right\|^{m}\left|x_{n}\right|^{-1}+\left\|u_{n}\right\|\left|x_{n}\right|^{k-2}\right)\left|x_{n}\right|^{1+\delta-2 \epsilon} \\
& +K_{2}^{\prime}\left(\left\|u_{n}\right\|^{m-1}+\left|x_{n}\right|^{k-1}\right)\|u\|\left|x_{n}\right|^{\delta-\epsilon} \\
& \leq C_{4} \sum_{n=0}^{\infty}\left|\frac{x_{n}}{x}\right|^{-\beta}\left(\left\|u_{n}\right\|^{m-1}+\left|x_{n}\right|^{k-1}\right)\left(K_{3}^{\prime}\left(\left\|u_{n}\right\|\left|x_{n}\right|^{2}\left|\log x_{n}\right|^{q}+\left\|u_{n}\right\|^{2}\left|x_{n}\right|\right)\right) \\
& +K_{1}^{\prime}\left(\left\|u_{n}\right\|\left|x_{n}\right|\left|\log x_{n}\right|^{q}+\left\|u_{n}\right\|^{2}\right)\left|x_{n}\right|^{1+\delta-2 \epsilon} \\
& +\left.K_{2}^{\prime}\left(\left|x_{n}\right|^{2}\left|\log x_{n}\right|^{q}+\left\|u_{n}\right\|\left|x_{n}\right|\right)\|u\||| x_{n}\right|^{\delta-\epsilon} \\
& \leq C_{5}^{\prime \prime \prime}\left(\|u\|^{m}+\left.\|u\||| x\right|^{k-1}\right) \text {, }
\end{aligned}
$$

where $C_{4}^{\prime \prime \prime}$ is a constant depending only on $H_{1}$ and where $C_{1}^{\prime \prime \prime}, C_{2}^{\prime \prime \prime}, C_{3}^{\prime \prime \prime}, C_{5}^{\prime \prime \prime}$ are small compared to $C_{4}^{\prime \prime \prime}$. Set

$$
K_{3}=2 C_{4}^{\prime \prime \prime}
$$


We then have

$$
\left\|\frac{\partial T \varphi}{\partial z}\right\| \leq K_{3}\left(\|u\|^{m}+\|u\||x|^{k-1}\right) .
$$

By definition, $K_{1}, K_{2}$ and $K_{3}$ are constants depending only on $H_{1}$. Therefore the proposition is true for these constants $K_{1}, K_{2}$ and $K_{3}$.

Let $B_{1}$ be the closed subset of $B_{0}$ defined by (3.3). Proposition 3.2 shows that the operator $T$ sends $B_{1}$ into itself. To finish the proof of Proposition 2.5, we have to show that $T$ restricted to $B_{1}$ is a contraction.

Let $\left(x_{n}, u_{n}, z_{n}\right)$ and $\left(x_{n}^{\prime}, u_{n}^{\prime}, z_{n}^{\prime}\right)$ be the iterates of $(x, u, z)$ by the transformations (2.9) associated to $\varphi$ and $\varphi^{\prime}$ respectively, where $\varphi, \varphi^{\prime} \in B_{1}$. Write $\Delta x_{n}$ for $\left|x_{n}-x_{n}^{\prime}\right|$, $\Delta u_{n}$ for $\left\|u_{n}-u_{n}^{\prime}\right\|, \Delta z_{n}$ for $\left\|z_{n}-z_{n}^{\prime}\right\|$ and $\Delta \varphi_{n}$ for $\left(\left\|u_{n}\right\|^{m}+\left\|u_{n}\right\|\left|x_{n}\right|^{k-1}\right)\left\|\varphi-\varphi^{\prime}\right\|_{0}$. When $n=0$, we will drop the subscript 0 .

We have the following

Lemma 3.3. For $\gamma, s, \rho, \tau$ small enough, we have the following inequalities in $D$ :

$$
\frac{\Delta x_{n}}{\Delta \varphi} \leq \frac{\left|x_{n}\right|^{1+\delta-\epsilon}}{|x|^{\delta}}, \quad \frac{\Delta u_{n}}{\Delta \varphi} \leq\left|\frac{x_{n}}{x}\right|^{\delta}, \quad \frac{\Delta z_{n}}{\Delta \varphi} \leq\left|\frac{x_{n}}{x}\right|^{\delta} .
$$

Proof. For $\gamma, s, \rho, \tau$ small enough, we have the following inequalities in $D$ :

$$
\begin{aligned}
& \frac{\Delta x_{1}}{\Delta \varphi} \leq K|x|^{2} \leq \frac{\left|x_{1}\right|^{1+\delta-\epsilon}}{|x|^{\delta}}, \\
& \frac{\Delta u_{1}}{\Delta \varphi} \leq K\left(|x|^{2}|\log x|^{q}+\|u\||x|\right) \leq\left|\frac{x_{1}}{x}\right|^{\delta}, \\
& \frac{\Delta z_{1}}{\Delta \varphi} \leq K\left(|x|^{2}+\|z\||| x \mid\right) \leq\left|\frac{x_{1}}{x}\right|^{\delta},
\end{aligned}
$$

where $K$ is a constant independent of $x, u, v, z$ and $q \in \mathbf{N}$.

Therefore (3.7) is true for $n=1$. We will show (3.7) by induction on $n$.

Using (2.3), we have

$$
\begin{aligned}
\Delta \varphi_{n} & =\left(\left\|u_{n}\right\|^{m}+\left\|u_{n}\right\|\left|x_{n}\right|^{k-1}\right)\left\|\varphi-\varphi^{\prime}\right\|_{0} \\
& \leq\left(\|u\|^{m}\left|\frac{x_{n}}{x}\right|^{m \alpha}+\|u\||x|^{k-1}\left|\frac{x_{n}}{x}\right|^{\alpha+k-1}\right)\left\|\varphi-\varphi^{\prime}\right\|_{0} \\
& \leq\left(\left|\frac{x_{n}}{x}\right|^{m \alpha}+\left|\frac{x_{n}}{x}\right|^{\alpha+k-1}\right)\left(\|u\|^{m}+\left.\|u\||| x\right|^{k-1}\right)\left\|\varphi-\varphi^{\prime}\right\|_{0} \\
& =\left(\left|\frac{x_{n}}{x}\right|^{m \alpha}+\left|\frac{x_{n}}{x}\right|^{\alpha+k-1}\right) \Delta \varphi .
\end{aligned}
$$

Since $\left|\frac{x_{n}}{x}\right|^{b} \leq\left|\frac{x_{n}}{x}\right|^{a}$ for $b>a$, we then have

$$
\frac{\Delta \varphi_{n}}{\Delta \varphi} \leq 2\left|\frac{x_{n}}{x}\right|^{\delta}
$$


Assume that (3.7) is true for $n$. Using (2.3), (3.2) and (3.8), we have

$$
\begin{aligned}
& \frac{\Delta x_{n+1}}{\Delta \varphi} \leq\left|1-2 x_{n}+o\left(x_{n}\right)\right| \frac{\Delta x_{n}}{\Delta \varphi}+K\left|x_{n}\right|^{2}\left(\frac{\Delta u_{n}}{\Delta \varphi}+\frac{\Delta \varphi_{n}}{\Delta \varphi}\right) \\
& +K\left(\left|x_{n}\right|^{3}\left|\log x_{n}\right|^{q}+\left\|u_{n}\right\|\left|x_{n}\right|^{2}\right) \frac{\Delta z_{n}}{\Delta \varphi} \\
& \leq \frac{\left|x_{n}\right|^{1+\delta-\epsilon}}{|x|^{\delta}}\left(\left|1-2 x_{n}+o\left(x_{n}\right)\right|+3 K\left|x_{n}\right|^{1+\epsilon}\right. \\
& \left.+K\left(\left|x_{n}\right|^{2+\epsilon}\left|\log x_{n}\right|^{q}+\left\|u_{n}\right\|\left|x_{n}\right|^{1+\epsilon}\right)\right) \\
& \leq \frac{\left|x_{n+1}\right|^{1+\delta-\epsilon}}{|x|^{\delta}}, \\
& \frac{\Delta u_{n+1}}{\Delta \varphi} \leq K\left\|u_{n}\right\| \frac{\Delta x_{n}}{\Delta \varphi}+\left|1-(\alpha+\epsilon) x_{n}\right| \frac{\Delta u_{n}}{\Delta \varphi}+K\left(\left|x_{n}\right|^{2}\left|\log x_{n}\right|^{q}+\left\|u_{n}\right\|\left|x_{n}\right|\right) \frac{\Delta \varphi_{n}}{\Delta \varphi} \\
& +K\left(\left\|u_{n}||\left|x_{n}\right|^{2}\left|\log x_{n}\right|^{q}+\right\| u_{n} \|^{2}\left|x_{n}\right|\right) \frac{\Delta z_{n}}{\Delta \varphi} \\
& \leq\left|\frac{x_{n}}{x}\right|^{\delta}\left(\left|1-(\alpha+\epsilon) x_{n}\right|+K \| u_{n}||\left|x_{n}\right|^{1-\epsilon}+2 K\left(\left|x_{n}\right|^{2}\left|\log x_{n}\right|^{q}+\left\|u_{n}\right\|\left|x_{n}\right|\right)\right. \\
& \left.+K\left(\left\|u_{n}\right\|\left|x_{n}\right|^{2}\left|\log x_{n}\right|^{q}+\left\|u_{n}\right\|^{2}\left|x_{n}\right|\right)\right) \\
& \leq\left|\frac{x_{n+1}}{x}\right|^{\delta}, \\
& \frac{\Delta z_{n+1}}{\Delta \varphi} \leq K\left(\left|x_{n}\right|+\left\|z_{n}\right\|\right) \frac{\Delta x_{n}}{\Delta \varphi}+K\left(\left|x_{n}\right|^{2}+\left\|z_{n}\right\|\left|x_{n}\right|\right)\left(\frac{\Delta u_{n}}{\Delta \varphi}+\frac{\Delta \varphi_{n}}{\Delta \varphi}\right) \\
& +\left(c+K\left|x_{n}\right|\right) \frac{\Delta z_{n}}{\Delta \varphi} \\
& \leq\left|\frac{x_{n}}{x}\right|^{\delta}\left(c+K\left|x_{n}\right|+K\left(\left|x_{n}\right|+\left\|z_{n}\right\|\right)+3 K\left(\left|x_{n}\right|^{2}+\left\|z_{n}\right\|\left|x_{n}\right|\right)\right) \\
& \leq\left|\frac{x_{n+1}}{x}\right| \delta \frac{c+K\left|x_{n}\right|+K\left(\left|x_{n}\right|+\left\|z_{n}\right\|\right)+3 K\left(\left|x_{n}\right|^{2}+\left\|z_{n}\right\|\left|x_{n}\right|\right)}{\left|1-(1+\epsilon) x_{n}\right|} \\
& \leq\left|\frac{x_{n+1}}{x}\right|^{\delta} \text {. }
\end{aligned}
$$

This proves the lemma.

We can write the difference

$S:=$

$\sum_{n=0}^{\infty}\left(\frac{x_{n}}{x}\right)^{-B} H_{1}\left(x_{n}, u_{n}, \varphi\left(x_{n}, u_{n}, z_{n}\right), z_{n}\right)-\sum_{n=0}^{\infty}\left(\frac{x_{n}^{\prime}}{x}\right)^{-B} H_{1}\left(x_{n}^{\prime}, u_{n}^{\prime}, \varphi^{\prime}\left(x_{n}^{\prime}, u_{n}^{\prime}, z_{n}^{\prime}\right), z_{n}^{\prime}\right)$

as

$$
S=S_{1}+S_{2}+S_{3},
$$

where

$$
\begin{aligned}
& S_{1}=\sum_{n=0}^{\infty}\left(\frac{x_{n}}{x}\right)^{-B}\left(H_{1}\left(x_{n}, u_{n}, \varphi\left(x_{n}, u_{n}, z_{n}\right), z_{n}\right)-H_{1}\left(x_{n}^{\prime}, u_{n}^{\prime}, \varphi\left(x_{n}^{\prime}, u_{n}^{\prime}, z_{n}^{\prime}\right), z_{n}^{\prime}\right)\right), \\
& S_{2}=\sum_{n=0}^{\infty}\left(\left(\frac{x_{n}}{x}\right)^{-B}-\left(\frac{x_{n}^{\prime}}{x}\right)^{-B}\right) H_{1}\left(x_{n}^{\prime}, u_{n}^{\prime}, \varphi\left(x_{n}^{\prime}, u_{n}^{\prime}, z_{n}^{\prime}\right), z_{n}^{\prime}\right), \\
& S_{3}=\sum_{n=0}^{\infty}\left(\frac{x_{n}^{\prime}}{x}\right)^{-B}\left(H_{1}\left(x_{n}^{\prime}, u_{n}^{\prime}, \varphi\left(x_{n}^{\prime}, u_{n}^{\prime}, z_{n}^{\prime}\right), z_{n}^{\prime}\right)-H_{1}\left(x_{n}^{\prime}, u_{n}^{\prime}, \varphi^{\prime}\left(x_{n}^{\prime}, u_{n}^{\prime}, z_{n}^{\prime}\right), z_{n}^{\prime}\right)\right) .
\end{aligned}
$$


Using (2.4), (3.3), (3.5), (3.6) and Lemma 3.3, we have

$$
\begin{aligned}
& \left\|S_{1}\right\| \leq \sum_{n=0}^{\infty}\left|\frac{x_{n}}{x}\right|^{-\beta}\left\{\left[C_{3}\left(\left\|u_{n}\right\|^{m}+\left\|u_{n}\right\|\left|x_{n}\right|^{k-1}\right)\right.\right. \\
& \left.+C_{4}\left(\left|x_{n}\right|^{2}\left|\log x_{n}\right|^{q}+\left\|u_{n}\right\|\left|x_{n}\right|\right) K_{1}\left(\left\|u_{n}\right\|^{m}\left|x_{n}\right|^{-1}+\left\|u_{n}\right\|\left|x_{n}\right|^{k-2}\right)\right] \Delta x_{n} \\
& +\left[C_{3}\left(\left\|u_{n}\right\|^{m-1}\left|x_{n}\right|+\left|x_{n}\right|^{k}\right)\right. \\
& \left.+C_{4}\left(\left|x_{n}\right|^{2}\left|\log x_{n}\right|^{q}+\| u_{n}||\left|x_{n}\right|\right) K_{2}\left(\left\|u_{n}\right\|^{m-1}+\left|x_{n}\right|^{k-1}\right)\right] \Delta u_{n} \\
& +\left[C_{3}\left(\left\|u_{n}\right\|^{m}\left|x_{n}\right|+\left\|u_{n}\right\|\left|x_{n}\right|^{k}\right)\right. \\
& \left.\left.+C_{4}\left(\left|x_{n}\right|^{2}\left|\log x_{n}\right|^{q}+\left\|u_{n}\right\|\left|x_{n}\right|\right) K_{3}\left(\left\|u_{n}\right\|^{m}+\left\|u_{n}\right\|\left|x_{n}\right|^{k-1}\right)\right] \Delta z_{n}\right\} \\
& \leq \sum_{n=0}^{\infty}\left|\frac{x_{n}}{x}\right|^{-\beta}\left\{( \| u _ { n } \| ^ { m } + \| u _ { n } \| | x _ { n } | ^ { k - 1 } ) \left[C_{3}\right.\right. \\
& \left.+C_{4} K_{1}\left(\left|x_{n}\right|\left|\log x_{n}\right|^{q}+\left\|u_{n}\right\|\right)\right] \frac{\left|x_{n}\right|^{1+\delta-\epsilon}}{|x|^{\delta}} \\
& +\left(\left\|u_{n}\right\|^{m-1}\left|x_{n}\right|+\left|x_{n}\right|^{k}\right)\left[C_{3}+C_{4} K_{2}\left(\left|x_{n}\right|\left|\log x_{n}\right|^{q}+\left\|u_{n}\right\|\right)\right]\left|\frac{x_{n}}{x}\right|^{\delta} \\
& +\left(\left\|u_{n}\right\|^{m}\left|x_{n}\right|+\left\|u_{n}\right\|\left|x_{n}\right|^{k}\right)\left[C_{3}+C_{4} K_{3}\left(\left|x_{n}\left\|\left.\log x_{n}\right|^{q}+\right\| u_{n} \|\right)\right]\left|\frac{x_{n}}{x}\right|^{\delta}\right\} \Delta \varphi \\
& \leq \sum_{n=0}^{\infty}\left|\frac{x_{n}}{x}\right|^{\delta-\beta}\left\{( \| u _ { n } \| ^ { m - 1 } | x _ { n } | + | x _ { n } | ^ { k } ) \left[C_{3}+C_{4} K_{2}\left(\left|x_{n}\right|\left|\log x_{n}\right|^{q}+\left\|u_{n}\right\|\right)\right.\right. \\
& +\left(C_{3}+C_{4} K_{1}\left(\left|x_{n}\right|\left|\log x_{n}\right|^{q}+\left\|u_{n}\right\|\right)\right)\left\|u_{n}\right\|\left|x_{n}\right|^{-\epsilon} \\
& \left.\left.+\left(C_{3}+C_{4} K_{3}\left(\left|x_{n}\right|\left|\log x_{n}\right|^{q}+\left\|u_{n}\right\|\right)\right)\left\|u_{n}\right\|\left|x_{n}\right|\right]\right\} \Delta \varphi \\
& \leq C_{5}\left(\|u\|^{m-1}+|x|^{k-1}\right)\left(\|u\|^{m}+\|u\||x|^{k-1}\right)\left\|\varphi-\varphi^{\prime}\right\|_{0} \text {, } \\
& \left\|S_{2}\right\| \leq \beta \sum_{n=0}^{\infty}\left|\frac{x_{n}}{x}\right|^{-\beta}\left(C_{1}+1\right)\left(\left\|u_{n}\right\|^{m}\left|x_{n}\right|+\| u_{n}||\left|x_{n}\right|^{k}\right)\left|x_{n}\right|^{-1} \Delta x_{n} \\
& \leq\left(C_{1}+1\right) \beta \sum_{n=0}^{\infty}\left|\frac{x_{n}}{x}\right|^{\delta-\beta}\left(\left\|u_{n}\right\|^{m-1}\left|x_{n}\right|+\left|x_{n}\right|^{k}\right)\left\|u_{n}\right\|\left|x_{n}\right|^{-\epsilon} \Delta \varphi \\
& \leq C_{6}\left(\|u\|^{m-1}+|x|^{k-1}\right)\left(\|u\|^{m}+\|u\||x|^{k-1}\right)\left\|\varphi-\varphi^{\prime}\right\|_{0}, \\
& \left\|S_{3}\right\| \leq \sum_{n=0}^{\infty}\left|\frac{x_{n}^{\prime}}{x}\right|^{-\beta} K\left(\left|x_{n}^{\prime}\right|^{2}\left|\log x_{n}^{\prime}\right|^{q}+\left\|u_{n}^{\prime}\right\||| x_{n}^{\prime} \mid\right) \Delta \varphi_{n}^{\prime} \\
& \leq C_{7}\left(|x||\log x|^{q}+\|u\|\right)\left(\|u\|^{m}+\|u\||x|^{k-1}\right)\left\|\varphi-\varphi^{\prime}\right\|_{0},
\end{aligned}
$$

where $C_{5}, C_{6}, C_{7}$ are constants depending only on $H_{1}$.

The above inequalities show that $T$ restricted to $B_{1}$ is a contraction. Therefore, the proof of Proposition 2.5 is complete.

\section{REFERENCES}

[F] Fatou, P.; Substitutions analytiques et équations fonctionnelles à deux variables, Ann. Sc. Ec. Norm. Sup. 40 (1924), 67-142.

[H1] Hakim, M.; Attracting domains for semi-attractive transformations of $\mathbf{C}^{p}$, Publ. Mat. 38 (1994), 479-499. MR1316642(96a:32050)

[H2] Hakim, M.; Analytic transformations of $\left(\mathbf{C}^{p}, 0\right)$ tangent to the identity, Duke Math. J. 92 (1998), 403-428. MR1612730 (99a:32036) 
[H3] Hakim, M.; Transformations tangent to the identity. Stable pieces of manifolds, Preprint, 1998.

[N] Nishimura, Y.; Automorphismes analytiques admettant des sous-variétés de points fixés attractives dans la direction transversale, J. Math. Kyoto Univ. 23 (1983), 289-299. MR706166 (85g:32040)

[Ri] Rivi, M.; Parabolic manifolds for semi-attractive holomorphic germs, Michigan Math. J. 49 (2001), 211-241. MR1852300 (2002k:32031)

[Ro] Rong, F.; Quasi-parabolic analytic transformations of $\mathbf{C}^{n}$. Parabolic manifolds, Ark. Mat. 48 (2010), no. 2, 361-370. MR2672615

[U1] Ueda, T.; Local structure of analytic transformations of two complex variables, I, J. Math. Kyoto Univ. 26 (1986), 233-261. MR849219 (87k:58145)

[U2] Ueda, T.; Local structure of analytic transformations of two complex variables, II, J. Math. Kyoto Univ. 31 (1991), 695-711. MR.1127094 (92i:58166)

Department of Mathematics, Syracuse University, Syracuse, New York 13244

Current address: Department of Mathematics, Shanghai Jiao Tong University, 800 Dong Chuan Road, Shanghai 200240, People's Republic of China 